Martha Cecilia Cueva; Amparo-de-Jesús Romero-Palacios; Nelly Germania Salguero-Barba;

Eliana Nathalie Palma-Corrales

http://dx.doi.org/10.35381/r.k.v6i11.1162

\title{
Capacitación dirigida a servidores públicos para la atención al cliente de personas con discapacidad
}

\section{Training aimed at public servants for customer service for people with disabilities}

\author{
Martha Cecilia Cueva \\ martha.cueva@utc.edu.ec \\ Universidad Técnica de Cotopaxi, Cotopaxi \\ Ecuador \\ https://orcid.org/0000-0003-2507-7285 \\ Amparo-de-Jesús Romero-Palacios \\ amparo.romero@utc.edu.ec \\ Universidad Técnica de Cotopaxi, Cotopaxi \\ Ecuador \\ https://orcid.org/0000-0003-2947-1626 \\ Nelly Germania Salguero-Barba \\ nelly.salguero@utc.edu.ec \\ Universidad Técnica de Cotopaxi, Cotopaxi \\ Ecuador \\ https://orcid.org/0000-0002-8866-4121 \\ Eliana Nathalie Palma-Corrales \\ eliana.palma@utc.edu.ec \\ Universidad Técnica de Cotopaxi, Cotopaxi \\ Ecuador \\ https://orcid.org/0000-0001-6515-5925
}

Recepción: 03 de octubre 2020

Revisado: 05 de noviembre 2020

Aprobación: 20 de diciembre 2020

Publicación: 01 de enero 2021 


\title{
RESUMEN
}

La investigación tiene por objetivo determinar la efectividad de un programa de capacitación dirigido a servidores públicos para la atención al cliente de personas con discapacidad auditiva y de habla en las empresas públicas del cantón Latacunga, Provincia de Cotopaxi - Ecuador. Se ejecutó desde una perspectiva metodológica explicativa con diseño cuasi experimental de pre y pos test, con grupo experimental control, aplicándose un tratamiento (programa de capacitación en atención al cliente), dirigido a 30 servidores públicos que laboran en las empresas públicas del cantón Latacunga, Provincia de Cotopaxi - Ecuador. Como principal conclusión se acepta H1 y se rechaza $\mathrm{HO}$, por lo tanto, el programa aplicado fue efectivo en la muestra poblacional estudiada, por lo tanto, la atención al cliente es un elemento fundamental en las empresas públicas, puede indicarse como la puerta de entrada del cliente, otro factor a tener en cuenta por parte del servidor público a la hora de prestar atención al cliente a una persona con discapacidad.

Descriptores: Psicopatología; marketing; empresa pública. (Palabras tomada del Tesauro UNESCO).

\begin{abstract}
The objective of the research is to determine the effectiveness of a training program aimed at public servants for customer service to people with hearing and speech disabilities in public companies in the Latacunga canton, Cotopaxi Province - Ecuador. It was executed from an explanatory methodological perspective with a quasi-experimental pre and posttest design, with an experimental - control group, applying a treatment (customer service training program), aimed at 30 public servants who work in public companies in the Latacunga canton. , Province of Cotopaxi - Ecuador. As the main conclusion, H1 is accepted and $\mathrm{HO}$ is rejected, therefore, the applied program was effective in the population sample studied, therefore, customer service is a fundamental element in public companies, it can be indicated as the gateway of the client, another factor to be taken into account by the public servant when providing customer service to a person with a disability.
\end{abstract}

Descriptors: Psychopathology; marketing; public enterprises. (Words taken from the UNESCO Thesaurus). 


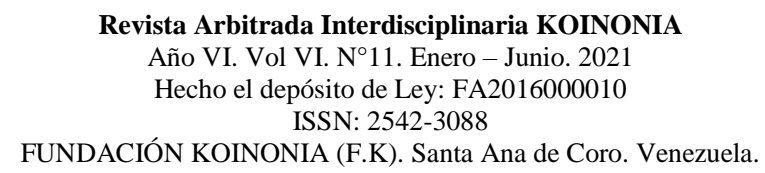

Martha Cecilia Cueva; Amparo-de-Jesús Romero-Palacios; Nelly Germania Salguero-Barba;

Eliana Nathalie Palma-Corrales

\section{INTRODUCCIÓN}

La atención al cliente es un factor esencial para la prestación de un servicio de calidad, tanto a clientes internos o externos, lo cual es implícito a cada persona, siendo parte de su cordialidad y educación (Eduarte-Ramírez,1999), es así que se constituye en un eje fundamental para el éxito de la organización, por cuanto, la atención al cliente se encuentra ligada a las expectativas por parte del cliente de recibir un trato favorable a sus inquietudes, posibilitándose la compra de un producto o servicio, en resumen, la atención al cliente, puede considerarse la puerta que abre o cierra los ingresos de la empresa, siendo menester mantener en una posición óptima para el cliente (Salazar-Yépez \& Cabrera-Vallejo, 2016).

En este sentido, la atención al cliente, a juicio de (Aldana \& Piña, 2017), genera fidelidad entre el cliente y la empresa, cuando se cumplen con las expectativas - perspectivas, se crea un vínculo de fidelidad, lo cual garantiza ingresos regulares, con ello la productividad tiende a ser favorable para mantener operatividad, rentabilidad, remuneración a los empleados, entre otros procesos propios de una organización empresarial, por lo tanto, se vincula con su filosofía organizacional, entre la cual, se debe tener en primer lugar al cliente tanto interno como externo (Martínez \& El Kadi, 2019).

Es así que, en los últimos años, las organizaciones empresariales tanto del sector público o privado, por cambios en las legislaciones donde se obliga a incorporar personas con discapacidad al campo laboral, han asumido la contratación de personas que padecen alguna discapacidad, siendo la atención al público o marketing online, uno de los de mayor ocupación, por cuanto las empresas a la hora de contratar se encuentran con el reto de que las personas con discapacidad, aparte de su condición, en ocasiones no fueron formadas en pertinencia por el sistema educativo (Forcada-Rojkín, 2015), el cual a su vez no estaba preparado para tal fin.

Por consiguiente, se constituye en un reto el asumir la formación permanente de las personas con discapacidad tanto en la educación formal y no formal, con la finalidad de que puedan integrarse con mayor efectividad al campo laboral, siendo esta una sentida 


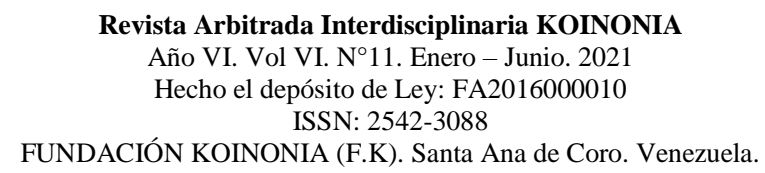

Martha Cecilia Cueva; Amparo-de-Jesús Romero-Palacios; Nelly Germania Salguero-Barba; Eliana Nathalie Palma-Corrales

necesidad que deben asumir las empresas, específicamente en el Ecuador, donde "el $85 \%$ de las personas con discapacidad no han accedido a la educación y solo el $15 \%$ ha terminado el colegio o tienen una profesión" (Sánchez-Ruiz, et al., 2019). Esta situación, obliga a los entes gubernamentales y privados, a trabajar en la formación por competencias de las personas con discapacidad, así como de los servidores públicos de atenderlos, de ese modo, podrán tener oportunidad de cumplir con las políticas internas de las empresas, especialmente si se trata en el área de atención al público.

Prosiguiendo con el caso ecuatoriano, (Bravo-González, et al., 2020), describen que la legislación en el Ecuador, es generosa para propiciar la integración de las personas con discapacidad al sistema educativo universitario, lo cual, redunda en lo laboral, sin embargo, cuestionan la escasa sensibilidad de las personas encargadas de las universidades de brindar mayor inclusión, siendo necesario un cambio de mentalidad, siendo esto recurrente al campo de trabajo, siendo necesario para contrarrestar estas acciones adversas, promover la formación permanente de las personas con discapacidad y por ende de los servidores púbicos, especialmente en el área de atención al cliente de las empresas públicas del Ecuador, con la finalidad de atender en calidad de servicio al segmento poblacional constituido por personas con discapacidad, se vean así, integrados efectivamente a la sociedad.

En razón de lo planteado, la investigación tiene por objetivo determinar la efectividad de un programa de capacitación dirigido a servidores públicos para la atención al cliente de personas con discapacidad auditiva y de habla en las empresas públicas del cantón Latacunga, Provincia de Cotopaxi - Ecuador.

\section{MÉTODO}

La investigación se ejecutó desde una perspectiva metodológica explicativa con diseño cuasi experimental de pre y pos test, con grupo experimental - control, aplicándose un tratamiento (programa de capacitación en atención al cliente), dirigido a 30 servidores públicos que laboran en las empresas públicas del cantón Latacunga, Provincia de 
Cotopaxi - Ecuador, los cuales pertenecieron al grupo experimental (G1), utilizándose una muestra control (G2) de 31 servidores públicos que no participaron directamente en el tratamiento.

Se aplicó una prueba en momento inicial a ambos grupos (G1-G2), luego se aplicó el tratamiento a G2, y una semana luego, se aplicó pos prueba a ambos grupos, para esto se empleó un instrumento tipo prueba que dictaminó el conocimiento que poseían las personas, el cual constaba de 16 ítems de varias alternativas de respuestas, validado por juicio de expertos y coeficiente Alfa de Cronbach con resultado de 0,87 siendo confiable para su aplicación.

Los datos obtenidos, se procesaron en razón de la prueba t de Student, lo cual permitió conocer la significancia bilateral y así, conocer el movimiento estadístico, para calificar si era efectivo o no, el programa aplicado, para este fin se empleó el programa estadístico IBM SPSS v 25.

\section{RESULTADOS}

En razón de la aplicación del programa de capacitación, se presentan los siguientes resultados: 
Tabla 1.

Capacitación en atención al público.

Prueba para una muestra

\begin{tabular}{|c|c|c|c|c|c|c|}
\hline \multicolumn{7}{|c|}{ Valor de prueba $=5$} \\
\hline & \multirow[t]{2}{*}{$\mathrm{T}$} & \multirow[t]{2}{*}{ gl } & \multirow[t]{2}{*}{$\begin{array}{l}\text { Sig. } \\
\text { (bilateral) }\end{array}$} & \multirow[t]{2}{*}{$\begin{array}{l}\text { Diferencia } \\
\text { de } \\
\text { medias }\end{array}$} & \multicolumn{2}{|c|}{$\begin{array}{c}95 \% \text { de intervalo de } \\
\text { confianza }\end{array}$} \\
\hline & & & & & Inferior & Superior \\
\hline G1PRETES & 31,165 & 30 & 0 & $-3,11537$ & $-3,3142$ & $-2,9163$ \\
\hline G2PRETES & 23,067 & 31 & $\underline{0}$ & $-3,0656$ & $-3,3304$ & $-2,802$ \\
\hline G1POSTEST & $-2,296$ & 30 & 0,023 & $-0,0643$ & $-0,1196$ & $-0,0084$ \\
\hline G2POSTEST & 63,951 & 31 & 0 & $-3,76317$ & $-3,8803$ & $-3,6458$ \\
\hline
\end{tabular}

Los resultados evidencian que existió modificación en G1 al momento de aplicar pos prueba, lo cual, implica modificación en el conocimiento de la muestra poblacional en comparación con G2 que no se recibió la capacitación, por lo tanto, se determina que el programa de capacitación aplicado, fue efectivo en la población estudiada.

\section{Prueba de hipótesis}

H1: La aplicación de un programa de capacitación de atención al cliente dirigido a servidores públicos, será efectivo para brindar una mejor atención a personas con discapacidad auditiva y de habla en las empresas públicas del cantón Latacunga, Provincia de Cotopaxi - Ecuador.

H0: La aplicación de un programa de capacitación de atención al cliente dirigido a servidores públicos, no será efectivo para brindar una mejor atención a personas con discapacidad auditiva y de habla en las empresas públicas del cantón Latacunga, Provincia de Cotopaxi - Ecuador. 


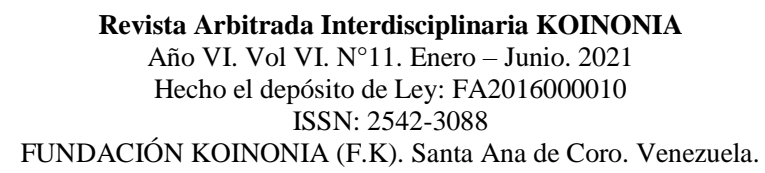

Martha Cecilia Cueva; Amparo-de-Jesús Romero-Palacios; Nelly Germania Salguero-Barba;

Eliana Nathalie Palma-Corrales

En función de los resultados obtenidos, se acepta $\mathrm{H} 1$ y se rechaza $\mathrm{H} 0$, por lo tanto, el programa aplicado fue efectivo en la muestra poblacional estudiada.

\section{DISCUSIÓN}

La atención al cliente es un elemento fundamental en las empresas públicas, puede indicarse como la puerta de entrada del cliente, lo cual tiene relación con la imagen corporativa de la organización, siendo esta, constituida por lo material e inmaterial, constituyéndose en la emocionalidad del cliente, promoviéndose una identidad con la empresa (Ramos-Farroñán \& Valle-Palomino, 2020), además cuando se trata de un cliente con discapacidad auditiva y de habla, intervienen factores como los derechos humanos, sensibilidad, ética, como elementos adicionales para brindar un servicio especial, lo cual es necesaria para cumplir con las expectativas y perspectivas del cliente, tal como indican (Aldana \& Piña, 2017).

Así mismo, el servidor público que atiende a la persona con discapacidad auditiva, debe tener en cuenta que esta puede padecer "mayores tasas de diabetes, enfermedades cardíacas, depresión, entre otras" (Campos \& Cartes-Velásquez, 2019, p. 639), esto involucra la posibilidad de sufrir cuadros de depresión, angustia, ansiedad, lo cual implica, brindar una atención preferencial de primer orden, por consiguiente es fundamental que el servidor público dedicado a la atención de este segmento poblacional, tenga sensibilidad, así como conocimientos básicos en medicina, psicología, con la finalidad de aplicar estrategias asertivas para evitar contratiempos o inconvenientes a la hora de establecer el abordaje con la persona con discapacidad auditiva y de habla.

Lo planteado, se complementa con lo expuesto por (Bravo-González, et al., 2020), al indicar que la legislación ecuatoriana favorece la atención preferencial de las personas con discapacidad, lo cual es amparado por la ley, fusionándose así, una atención adecuada por ser cliente, pero además, con disposiciones sanitarias que subyacen en su actuación en un determinado momento, como lo es, ser atendido en una empresa pública, así mismo, otro factor a tener en cuenta por parte del servidor público a la hora de prestar 
atención al cliente a una persona con discapacidad, es la posible escasa formación educativa que posea, tal como plantea (Sánchez-Ruiz, et al., 2019), lo cual involucra además de la propia condición de la persona, su salud integral y educación.

En este sentido, es aconsejable seguir la recomendación de (Palmeros-y-Ávila \& GairínSallán, 2016), de aplicar un acompañamiento tutorial a las personas con discapacidad para que sean integradas con mayor efectividad a la educación universitaria, sin embargo, esta situación puede extenderse a los servidores públicos, con la finalidad de que progresivamente obtengan formación que les permita atender con mayor efectividad a las personas con discapacidad, siendo una estrategia que podría aplicarse para acompañar al cliente con discapacidad, hasta que obtenga respuesta favorable y así, perciba una atención empática, afable, favorable para tener una visión afirmativa de la empresa pública, siendo este uno de los pilares de la atención al cliente, tal como lo describen (Martínez \& El Kadi, 2019), posibilitando así, un marketing favorable sobre la organización y su transparencia al atender al cliente con discapacidad (Forcada-Rojkín, 2015). En la figura 1, se evidencian los tipos de discapacidad en el Ecuador:

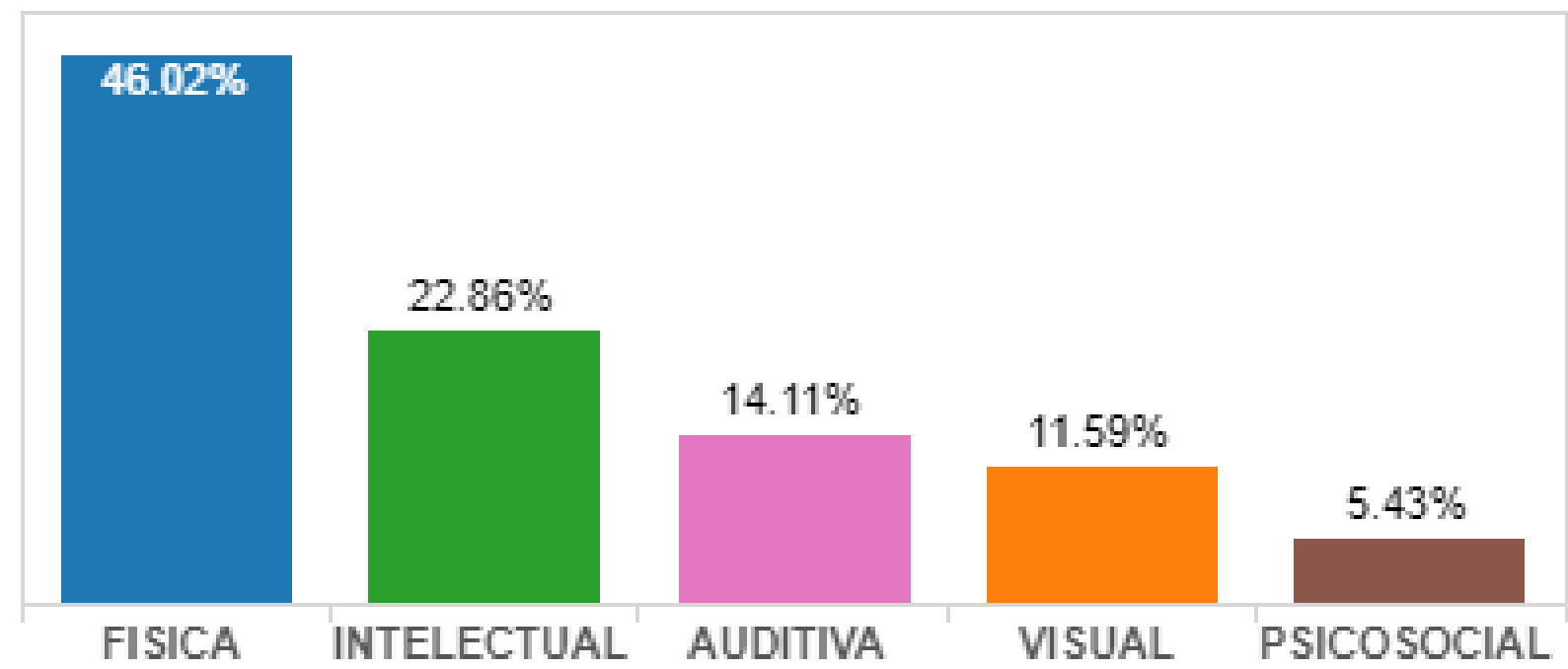

Figura 1. Tipos de discapacidad. Fuente: Consejo nacional para la igualdad de discapacidades (2021). 
La discapacidad auditiva representa el $14,11 \%$ de la población discapacitada, esto en cierto modo es positivo por no ser un segmento proporcional, lo cual brinda oportunidad de formar a los servidores públicos progresivamente, así puedan establecer una atención al cliente favorable, siendo esto un indicador que es posible crecer socialmente en razón de establecer un principio de reciprocidad de empatía entre las personas, así se genera una sociedad basada en el respeto mutuo y equidad social, generándose una imagen positiva del servidor público en prestación de servicios al cliente.

\section{CONCLUSIÓN}

Como principal conclusión se acepta $\mathrm{H} 1$ y se rechaza $\mathrm{H} 0$, por lo tanto, el programa aplicado fue efectivo en la muestra poblacional estudiada, por lo tanto, la atención al cliente es un elemento fundamental en las empresas públicas, puede indicarse como la puerta de entrada del cliente, otro factor a tener en cuenta por parte del servidor público a la hora de prestar atención al cliente a una persona con discapacidad, es la posible escasa formación educativa que posea, lo cual, involucra la posibilidad de sufrir cuadros de depresión, angustia, ansiedad, lo cual implica, brindar una atención preferencial de primer orden, por consiguiente es fundamental que el servidor público dedicado a la atención de este segmento poblacional, tenga sensibilidad, así como conocimientos básicos en medicina, psicología, con la finalidad de aplicar estrategias asertivas para evitar contratiempos o inconvenientes a la hora de establecer el abordaje con la persona con discapacidad auditiva y de habla.

\section{FINANCIAMIENTO}

No monetario.

\section{AGRADECIMIENTO}

A la Universidad Técnica de Cotopaxi; por motivar el desarrollo de la investigación. 


\section{REFERENCIAS CONSULTADAS}

Aldana, J., \& Piña, J. (2017). Calidad del servicio prestado al cliente por los instructores de gimnasios [Quality of customer service provided by gym instructors]. Revista Arbitrada Interdisciplinaria Koinonía, 2(3), 172-197.

Bravo-González, A, Córdova-Granda, J, \& Ramón-Merchán, M. (2020). La inclusión en la enseñanza superior de las personas discapacitadas en la legislación ecuatoriana [The inclusion of disabled persons in the superior education under the Ecuadorian legislation]. Conrado, 16(73), 327-334.

Campos, V, \& Cartes-Velásquez, R. (2019). Estado actual de la atención sanitaria de personas con discapacidad auditiva y visual: una revisión breve [Health care of people with visual or hearing disabilities]. Revista médica de Chile, 147(5), 634642. https://dx.doi.org/10.4067/S0034-98872019000500634

Consejo nacional para la igualdad de discapacidades (2021). Tipos de discapacidad [Types of disability]. Recuperado de https://n9.cl/rof9

Eduarte-Ramírez, A. (1999). Calidad en el servicio al cliente [Quality in customer service]. Revistas de Ciencias Administrativas y Financieras de la Seguridad Social, $7(1), 41-44$.

Forcada-Rojkín, C. (2015). La inserción de personas con discapacidad en el mercado laboral privado [The insertion of people with disabilities in the private labor market]. Invenio, 18(35),85-104.

Martínez, L., \& El Kadi, O. (2019). Logística Integral y Calidad Total, Filosofía de Gestión Organizacional orientadas al cliente [Comprehensive Logistics and Total Quality, Customer-oriented Organizational Management Philosophy] Revista Arbitrada Interdisciplinaria Koinonía, 4(7), 202-232. http://dx.doi.org/10.35381/r.k.v4i7.201

Palmeros-y-Ávila, G, \& Gairín-Sallán, J. (2016). La atención a las personas con discapacidad en las universidades mexicanas y españolas, desde la revisión de las políticas educativas [The care of people with disabilities in Mexican and Spanish universities]. Educación, 25(49), 83102. https://dx.doi.org/10.18800/educacion.201602.005 
Revista Arbitrada Interdisciplinaria KOINONIA

Año VI. Vol VI. N¹1. Enero - Junio. 2021

Hecho el depósito de Ley: FA2016000010

ISSN: 2542-3088

FUNDACIÓN KOINONIA (F.K). Santa Ana de Coro. Venezuela.

Martha Cecilia Cueva; Amparo-de-Jesús Romero-Palacios; Nelly Germania Salguero-Barba;

Eliana Nathalie Palma-Corrales

Ramos-Farroñán, E, \& Valle-Palomino, N. (2020). Gestión de imagen corporativa como estrategia de sostenibilidad: camino al cambio empresarial [Corporate image management as a sustainability strategy: path to business change]. Revista Universidad y Sociedad, 12(1), 292-298.

Salazar-Yépez, W, \& Cabrera-Vallejo, M. (2016). Diagnóstico de la calidad de servicio, en la atención al cliente, en la Universidad Nacional de Chimborazo - Ecuador [Diagnosis of the quality of service, in customer service, at the National University of Chimborazo - Ecuador]. Industrial Data, 19(2),13-20.

Sánchez-Ruiz, J, Román-Aguirre, R, Torres-Sánchez, A, \& Loarte-Tene, M. (2019). Inserción laboral y desarrollo profesional de las personas con discapacidades en Empresas Turísticas de la ciudad de Loja [Labor insertion and professional development of people with disabilities in Tourist Companies in the city of Loja]. Revista Universidad y Sociedad, 11(5), 201-205.

C2021 por los autores. Este artículo es de acceso abierto y distribuido según los términos y condiciones de la licencia Creative Commons Atribución-NoComercial-Compartirlgual 4.0 Internacional (CC BY-NC-SA 4.0)

(https://creativecommons.org/licenses/by-nc-sa/4.0/). 